\title{
Molecular architecture of SARS-CoV-2 envelope by integrative modeling
}

\author{
Weria Pezeshkian $^{1, \$}$, Fabian Grünewald ${ }^{1, \$}$, Oleksandr Narykov ${ }^{2, \$}$, Senbao Lu ${ }^{3}$, Tsjerk A \\ Wassenaar $^{1,4}$, Siewert J. Marrink ${ }^{1, *}$, and Dmitry Korkin ${ }^{2,3, *}$
}

\author{
Affiliations: \\ ${ }^{1}$ Groningen Biomolecular Sciences and Biotechnology Institute and Zernike Institute for \\ Advanced Materials, University of Groningen; 9747AG Groningen, The Netherlands. \\ ${ }^{2}$ Department of Computer Science, Worcester Polytechnic Institute; Worcester, MA, \\ USA \\ ${ }^{3}$ Bioinformatics and Computational Biology Program, Worcester Polytechnic Institute; \\ Worcester, MA, USA \\ ${ }^{4}$ Institute for Life Science and Technology, Hanze University of Applied Sciences; \\ 9747AS Groningen, The Netherlands \\ ${ }^{\$}$ These authors contributed equally to this work. \\ *Corresponding authors. Email: korkin@korkinlab.org (D.K.); s.j.marrink@rug.nl \\ (S.J.M.);
}

\begin{abstract}
Despite tremendous efforts by research community during the COVID-19 pandemic, the exact structure of SARS-CoV-2 and related betacoronaviruses remains elusive. Here, we developed and applied an integrative multi-scale computational approach to model the envelope structure of SARS-CoV-2, focusing on studying the dynamic nature and molecular interactions of its most abundant, but largely understudied, M (membrane) protein. The molecular dynamics simulations allowed us to test the envelop stability under different configurations and revealed that $\mathrm{M}$ dimers agglomerated into large, filament-like, macromolecular assemblies with distinct molecular patterns formed by M's transmembrane and intra-virion (endo) domains. These results were in agreement with the experimental data, demonstrating a generic and versatile integrative approach to model the structure of a virus de novo, providing insights into critical roles of structural proteins in the viral assembly and integration, and proposing new targets for the antiviral therapies.
\end{abstract}


Betacoronaviruses are enveloped positive-strand RNA viruses. It has been nearly 20 years since the first outbreak of a pathogenic betacoronavirus, SARS-CoV, which was followed by MERS-CoV, and, most recently, SARS-CoV-2 ${ }^{1-3}$. Yet, despite tremendous efforts in the elucidation of structural details of the viral proteins and protein complexes, a detailed virion structure of a betacoronavirus remains unsolved, due to the complexity and plasticity of these viruses. The virion particles of SARS-CoV-2 and closely related viruses are formed by four structural proteins: the spike $(\mathrm{S})$, membrane $(\mathrm{M})$, envelope $(\mathrm{E})$, and nucleocapsid $(\mathrm{N})$ proteins ${ }^{4}$. The first three of these proteins oligomerize, and together with lipids from the host cell membranes, form the viral envelope, while the function of $\mathrm{N}$ protein is to organize, pack, and protect the viral RNA strand. Detailed structural knowledge of the viral envelope is critical because it allows for a mechanistic understanding of interactions between the virus and the host cell, and because the envelope surface presents potential drug targets for therapeutic interventions 5 .

Electron microscopy and tomography studies of several betacoronaviruses, including SARS-CoV-2, have revealed that the morphology of the viral envelope is conserved while allowing certain flexibility in its overall shape ${ }^{6,7}$. The studies also suggest that the envelope forms an ellipsoid with the average diameters recently estimated for SARSCoV-2 to range between 53-77 nm, 77-95nm, and 85-109nm, respectively; comparable sizes have been previously reported for SARS-CoV ${ }^{8}$. The architecture of the envelope was estimated to include $26 \pm 15 \mathrm{~S}$ trimers ${ }^{6}$, which is less than the previously reported number of trimers in a closely related mouse hepatitis virus, MHV (average is $74 \mathrm{~S}$ trimers ${ }^{8}$ ). The number of $\mathrm{M}$ dimers in SARS-CoV-2 is currently unknown but is expected to be comparable to $\sim 1,100 \mathrm{M}$ dimers estimated on average for three other betacoronaviruses, SARS-CoV, MHV, and FCoV envelopes ${ }^{8}$. Lastly, E pentamers are estimated to localize in handful numbers in the envelopes of the above coronaviruses, including SARS-CoV-2 ${ }^{9-11}$. Being the most abundant protein of the envelope, M protein is integrated into the virus' lipid bilayer in a homodimeric form and plays an important yet not fully understood structural role. For instance, it is still unclear whether $M$ is sufficient to form a stable envelope structure in coronaviruses, or $\mathrm{S}$ and $\mathrm{E}$ are also required ${ }^{12}$. The role and nature of the interactions between these three proteins were suggested to be more complex than originally expected ${ }^{13}$. More recent work based on electron microscopy studies revealed the ability of $M$ dimers to form lattice-like structures and highlighted the role of the endodomain of $\mathrm{M}$ in lattice formation and interactions with other structural proteins, including $\mathrm{N}^{8,14}$.

Two recent electron microscopy and electron tomography studies of the virion particles of SARS-CoV-2 ${ }^{6,7}$ together with earlier microscopy studies of SARS-CoV and MHV ${ }^{8,15}$ have provided important information on the morphology of the virion, including basic local and global geometric patterns formed by the structural proteins on the envelope surface, the stoichiometry of the structural proteins contributing to the envelope, and the distribution of S-trimers. However, the details are obtained from averages of hundreds of images and thus provide more general, low-resolution, information on the spatial arrangement of the structural proteins. The main reason behind the lack of highresolution imaging data for any coronavirus is the flexible nature of the viral envelope, which prevents constructing models by fitting structures or structural models of proteins 
and lipids into the electron density. Thus, a de novo approach is required that does not rely on the density data ${ }^{16}$. Another challenge for the modeling is the lack of symmetry in the envelope, which is a typical feature of viral capsids. In addition, none of the three structural proteins constituting the viral envelope, E, M, or S, have been resolved experimentally in their full lengths for SARS-CoV-2. A full-length model of the spike trimer was recently constructed by combining high-resolution cryoelectron microscopy (Cryo-EM) data with the modeled structures of experimentally unsolved domains to create a full-length model ${ }^{17}$. A homology model of E pentamer was recently obtained ${ }^{18}$, followed by refinement in a lipid bilayer ${ }^{19}$. However, no accurate model of the M dimer currently exists - a de novo model of the two-domain monomer could not be corroborated by additional evidence ${ }^{8}$ and may contain substantial errors.

We developed an integrative approach to generate detailed models of the SARS-CoV-2 envelope by combining structural data from experiments and from homology models for the individual proteins, their oligomeric conformations, lipid bilayer composition, protein stoichiometries, the local geometries of the protein configurations, and the global geometry of the envelope (Fig. 1, Supp. Fig S1, Methods). In our approach, available EM data was used only to obtain information on local geometry to refine the structure of the M-dimer (Supp. Figs. S2, S3, Methods) and to set up the initial positions of the structural proteins in the envelope structures (Methods, Supp. Fig. S1). The global geometric patterns observed in the EM studies were not used during modeling, but only to evaluate our model (Methods, Fig. 4A-C). The integrative computational modeling pipeline includes five stages (Fig. 1): 1) structural characterization of S, M, and E proteins in both monomeric and homooligomeric forms; 2) lipid fingerprinting analysis; 3) construction of an initial structural configuration of the envelope by taking as an input the refined structures of the three homooligomers, their stoichiometry, composition of the lipid bilayer, as well as the geometry and size of the envelope; 4) coarse-grain (CG) molecular dynamics (MD) simulation; and 5) the analysis of the obtained structural trajectories.

The initial stage of modeling the entire envelope consisted of a dynamic triangulated surface (DTS) simulation ${ }^{20,21}$ to obtain a triangulated envelope shape matching the dimensions of the virion. This model was subsequently converted to a CG representation ${ }^{22}$, placing CG models of the proteins and lipids based on Martini force field model ${ }^{23}$ with specified stoichiometries (Supp. Fig. S1). This resulted in an initial arrangement of the oligomeric protein structures embedded in a lipid bilayer comprising six types of lipid molecules, with a composition reflecting that of the endoplasmic reticulum, but also considering enrichment of specific lipids due to interactions with the proteins, based on the lipid fingerprinting analysis (Methods). Several envelope models were thus built and subjected to MD simulations to test the stability of the envelope structure with different concentrations of lipids, different stoichiometries, and different simulation settings. Simulations for stable candidate models with the final selection of parameters were run for $4 \mu \mathrm{s}$. Finally, an all-atom model was obtained using the backward tool ${ }^{24}$ on the final CG model from the longest simulation.

The CG representations of the homooligomers of the structural proteins were obtained from available atomic models ( $\mathrm{S}$ and $\mathrm{E}$ ) and from de novo modeling (M) (Fig. 2C), which 
were coarse-grained and then refined in the presence of a lipid bilayer (Methods). The initial model of the full-length $\mathrm{S}$ trimer was obtained previously using an integrative modeling approach ${ }^{17}$, and a model of the E pentamer was obtained previously using homology modeling ${ }^{18,19}$. In contrast to the $\mathrm{S}$ and $\mathrm{E}$ proteins, a structure of $\mathrm{M}$ or its homodimer supported by experimental observations or evolutionary inference did not exist. Therefore, we first modeled the structure of the $\mathrm{M}$ dimer using an integrative approach (Supp. Figs. S2). The procedure started with the de novo modeling of the monomeric structure of $\mathrm{M}$, followed by constraint symmetric docking to create a homodimer that satisfies the geometric constraints obtained based on the envelope's membrane thickness, mutual orientation of the monomers, and the overall geometric boundaries of the $\mathrm{M}$ dimer complex previously obtained from microscopy data of the SARS-CoV envelope ${ }^{8}$. We found that the de novo homodimer models of M appeared to share striking structural similarity with another recently resolved homodimer of SARSCoV-2, the ORF3a protein ${ }^{25}$. The similarity included the same two-domain fold composition and the same combination of secondary structure elements as in our de novo model, but with a slightly different arrangement of the secondary structure elements in the transmembrane domain (Supp. Fig. S3). We thus further refined the M dimer model by constructing a new structural template as a scaffold of the same secondary structure elements as in the original de novo model, each of which was structurally superposed against the ORF3a dimer. We then applied a novel integrative template-modeling protocol using the newly designed template and followed by a refinement protocol guided by the electron density of ORF3a (Methods, Supp. Fig. S4). The resulting model not only provided a tighter, more stable packing of $\mathrm{M}$ monomers in the dimer; but the shape complementarity of $\mathrm{M}$ dimers with each other allowed for a natural tiling of multiple dimers into the "filament" structures, supporting the previously proposed model of Mdimer lattices based on the microscopy study of SARS-CoV envelope ${ }^{8,26}$.

The overall models prepared for the simulation consisted of 20-30 million CG particles. The models varied in several key parameters: (i) different stoichiometries of the structural proteins, (ii) full or partial ectodomains of the spike trimer included in the envelope structure, and (iii) different lipid-to-protein ratios. In total, 6 independent simulations were executed, two of which turned out to be unstable. The selected stable models, simulated for $1-4 \mu \mathrm{s}$, included $\sim 1.0-1.2 \mathrm{M}$ protein particles, $\sim 0.5-0.8 \mathrm{M}$ lipid particles, and $\sim 13 \mathrm{M}-35 \mathrm{M}$ solvent particles (Fig. 2A,B, Tables 1,2). A $4 \mu$ s simulation took $\sim 1,560,000$ CPU/hours to compute on TACC Frontera supercomputer. We found that each of the analyzed parameters played a key role in the simulation. First, when selecting between two different protein-to-lipids ratios, the higher ratio value of 2.36 resulted in an unstable structure, while the ratio of 1.44 resulted in a structural model that remained stable. Second, we found that varying the stoichiometries of E pentamer, S trimer, and M dimer under the same conditions does not affect the envelope stability: for both oligomeric compositions that included a truncated form of $\mathrm{S}$ trimer, $\mathrm{C} 1=(2 \mathrm{E}, 25 \mathrm{~S}, 1003 \mathrm{M})$ and $\mathrm{C} 2=(3 \mathrm{E}, 71 \mathrm{~S}, 1080 \mathrm{M})$, we were able to obtain stable $4 \mu$ s trajectories (Fig 2A,B, Supp. Fig. S5). The results suggested that similar molecular mechanisms underlie the structural integrity of the envelope for SARS-CoV-2, SARS-CoV, MHV, and other betacoronaviruses. The behavior of the envelope model that included the full-length $\mathrm{S}$ trimers and composition $\mathrm{C} 1$ was similar to the ones of the truncated model (Fig. 2A,B, 3B): a $1 \mu \mathrm{s}$ 
trajectory of the former was comparable to the first $1 \mu$ s of the $4 \mu$ s trajectory for the latter.

In the $4 \mu$ s simulations, we consistently observed changes in the viral shape (Fig. 2D, Supp. Figs. S6, S7), with the initial diameters of the ellipsoid of a model in composition C1 changing from $d_{1} \approx 109.9 \mathrm{~nm}, d_{2} \approx 97.8 \mathrm{~nm}$, and $d_{3} \approx 76.2$ at $\mathrm{t}=0 \mu$ s to $d_{1} \approx 103.1 \mathrm{~nm}$, $d_{2} \approx 97.8 \mathrm{~nm}$, and $d_{3} \approx 81.3 \mathrm{~nm}$ at the end of the simulation, $\mathrm{t}=4 \mu \mathrm{s}$. The obtained diameters were close to the range observed for the particles from the cryoelectron tomography (Cryo-ET) images of SARS-CoV-2 ${ }^{27}$. The calculated $d_{\mathrm{MAX}} / d_{\mathrm{MIN}}$ ratio of 1.27 for our final model was higher than the one previously observed in the ellipsoid structure of MHV virion $\left(d_{\mathrm{MAX}} / d_{\mathrm{MIN}}=1.08\right)^{8,15}$. Interestingly, the changes in the shape did not have significant effects on the surface area of the envelope (1.3\% reduction) or its volume (0.6\%) (Supp. Figs. S6, S7). Along with the diameters of the envelope shape, the principal radii of gyration were also converging, reflecting shape stabilization (Fig. 2D). Furthermore, analysis of the temporal changes of the viral dimensions together with the connectivity patterns of the envelope proteins suggested the presence of two distinct concurrent relaxation processes, separately affecting the two smallest and the two largest diameters. Specifically, we observed a faster process $(0-1 \mu \mathrm{s})$, followed by a slower process $(0.5-4 \mu \mathrm{s})$. During the faster process, the minor circumference (principal radii $r_{2}$ and $r_{3}$ corresponding to diameters $d_{2}$ and $d_{3}$ ) became more circular while during the slower process, the major circumference (principal radii $r_{1}$ and $r_{2}$ corresponding to diameters $d_{1}$ and $d_{2}$ ) also became more circular, thus making the minor circumference to become more elliptical again (Fig. 2D).

Our envelope models and the time scales of the simulations allowed a detailed assessment of the interactions between the different constituents, in particular those involving M-dimers. To characterize these interactions, we focused on the preferential relative orientations of protein neighbors and second neighbors (Fig. 4A-C), which were determined using a method for orientation analysis ${ }^{28}$. The results showed that the $\mathrm{M}$ dimer transmembrane domains (TMDs) preferentially formed filament-like assemblies, without contacts between adjacent filaments (Figs. 2A,B). In contrast, the endodomains (EDs) appear tightly packed, binding neighbors in two directions (Figs. 2A,B), thus showing a tendency for the formation of a well-ordered lattice. Intriguingly, the lattice vectors were identical to the one extracted from the previously reported EM data ${ }^{8}$. Combining the (averaged) relative orientations of the TMDs and the EDs with the projected densities of the proteins revealed a characteristic pattern of densities that fit almost exactly with the 'lattice' patterns previously observed from the averaged CryoEM virion structures of SARS-CoV and related betacoronaviruses (Fig. 4A,B) ${ }^{8}$, despite the fact that this information was not used during construction of the models. Even the characteristic lack of density in the unit cells' corners previously observed in MHV and SARS-CoV was clearly noticeable in our data (Fig. 4B). These patterns were consistent in the models with both compositions, $\mathrm{C} 1$ and $\mathrm{C} 2$. In contrast to the strong preferential orientation of $\mathrm{M}$ dimers, the orientation of $\mathrm{M}$ dimers around $\mathrm{S}$ trimers did not show clear preference in attachment (Fig. $4 \mathrm{C}$ ), which could be due to the stronger orienting effects of $\mathrm{M}$ dimer interactions as well as to the worse statistics for the interactions between $\mathrm{S}$ and $\mathrm{M}$ (Fig. 3B). 
To further characterize formation of the higher-order assemblies in the envelope through interactions between M-dimers, a temporal analysis was performed of the domain-level physical interaction networks between the TMDs and EDs of $\mathrm{M}$ dimers and the transmembrane domains of $\mathrm{S}$ trimers. This analysis further illustrated the strikingly different nature of the M dimer's key domains (Fig. 4D-G, Supp. Fig S8-S11), supporting our previous findings. Throughout the simulations, the domain interaction networks appeared to undergo drastic rearrangement via two distinct phases, with the number of connected components of 3 and more dimers first rapidly rising during 0-200ns up to $\sim 160$ components and then slowly saturating to $\sim 30$ components (Fig. 4D). The total number of connected components closely follows a bi-exponential law (Suppl. Figs. S8, S9), suggesting two processes running concurrently: a faster local rearrangement and a slower filament assembly. The two-process formation of the connected components was also evident from the clustering analysis (Fig. 4F), indicating the initial formation of many small clusters, followed by the preferential growth of the largest connected components. It was also interesting to see that the second, slower process of growing connected components started before the first, faster stage of forming initial small assemblies of $\mathrm{M}$ dimers was over. The analysis of the interaction network of TMDs also supported the formation of filaments observed on the surface of the envelope (Fig. 4E); the network revealed that these filaments were occasionally connected even further into larger assemblies. In contrast to the filament-like network topology, the EDs network consisted of connected triangulated components, which may contribute to the structural rigidity of the envelope. The difference between the TMD and ED network topologies also followed from the temporal node degree distributions for each network: the average node degrees converge to $\sim 2$ in TMD network and $\sim 3$ in ED network (Fig. 4G). Lastly, all other major network parameters appeared to converge to the stable values (Suppl. Figs. S8-S11), thus further drastic changes in network topologies were not expected.

Despite remarkable similarity of the virion structures shared between betacoronaviruses, such as SARS-CoV, MHV, and SARS-CoV-2, and the efforts to elucidate the structure of these viruses using imaging and computational methods ${ }^{26,27,29-31}$, no high-resolution experimental structure of the envelope currently exists. Our integrative approach allows combining experimental information at different resolutions into a consistent model, providing structural and functional insights beyond what can be obtained by a single experimental method. The obtained model is an important step towards our understanding of the underlying molecular architecture of the entire virus and successfully bridges the gap between molecular simulations and electron microscopy of virions, reproducing the experimentally observed density profiles of the local envelope structure for the first time. Furthermore, the developed, largely automated computational protocol can be readily applied to study the envelopes of other coronaviruses, once the models or experimental structures and stoichiometries of the structural proteins comprising the envelope are obtained. The model can serve as a structural scaffold for understanding the interplay between mutual orientations of neighbor spike trimers and the role of this orientation in the viral interaction with the host receptors, as well as for studying the interactions between the proposed elongated form of $\mathrm{M}$-dimers and $\mathrm{N}$-proteins ${ }^{8}$ to discern the mechanistic determinants of the virion's stability. The structures of M-dimers and the 
complex inter-dimeric assemblies they form can provide the structural basis for understanding the molecular mechanisms behind the viral assembly. The structural knowledge of complexes formed by $\mathrm{M}$ proteins can also be helpful when designing new antiviral compounds targeting the interaction interface of the $M$ protein and thus preventing formation of the envelope, an approach recently suggested for other viruses 32,33. The computational protocol developed as a part of this study can be readily applied to other emerging coronaviruses to provide rapid advancements in our understanding of the role of structural proteins. Finally, the structural model of the viral envelope will facilitate the development of viral-like nanoparticles for novel vaccines ${ }^{34}$.

Acknowledgments: We would like to thank Frank Alber, Sai Ganesan, Andrej Sali, Kuang Shen, and Celia Schiffer for the useful feedback about the manuscript.

Author contributions: All authors contributed extensively to the work presented in this paper. DK jointly conceived the study with SJM. WP, FG, ON, SL, TAW, and DK developed the methodology. WP, FG, ON, SL, and TAW performed the experiments and collected data. WP, FG, ON, SL, TAW, SJM, and DK analyzed data. WP, FG, ON, SL, TAW, and DK generated visualizations for different parts of the project. ON, SL, WP, TAW, and DK wrote the original draft, and all authors contributed to the review and editing of the manuscript. SJM and DK supervised and managed the project.

Competing interests: The authors declare no competing interests.

Materials \& Correspondence. Correspondence and material requests should be addressed to korkin@korkinlab.org (D.K.) and s.j.marrink@ rug.nl (S.J.M.). 


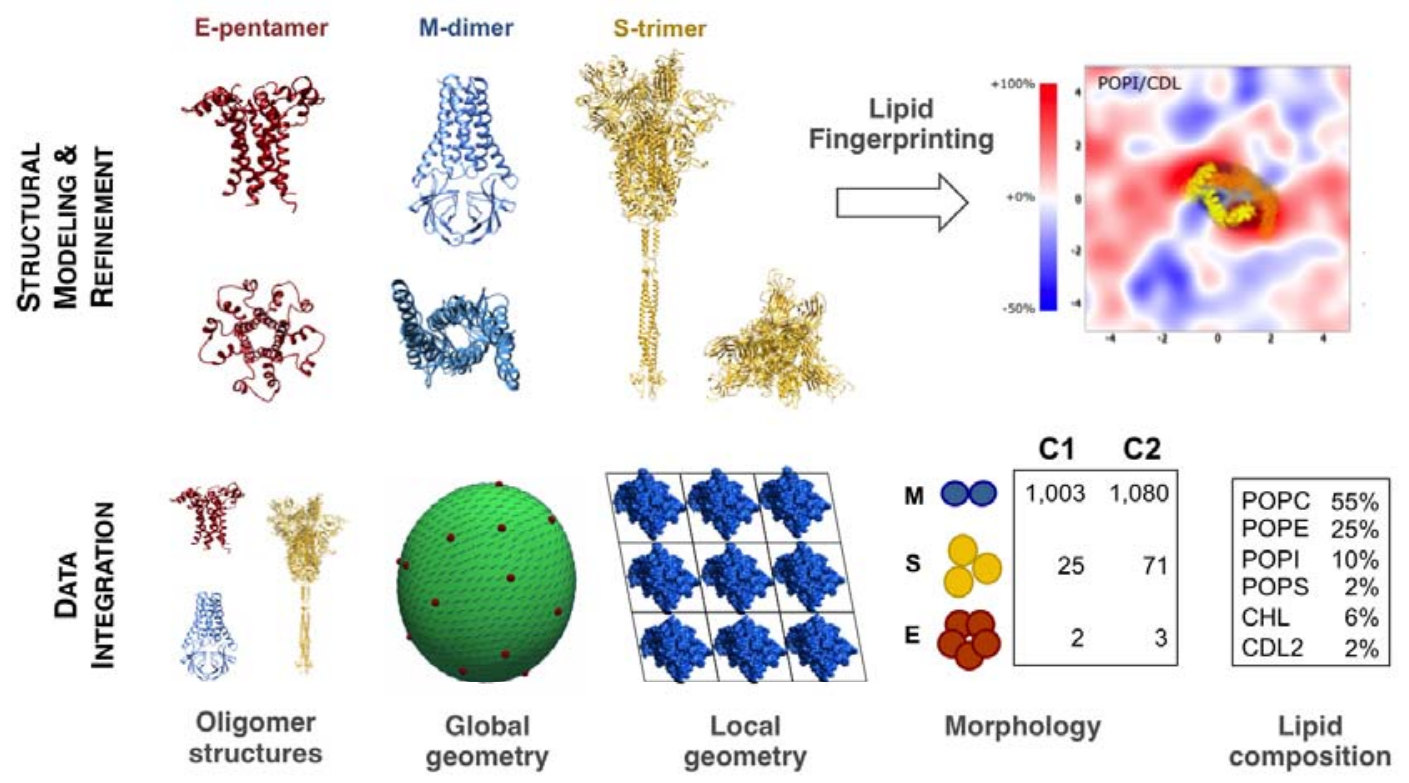

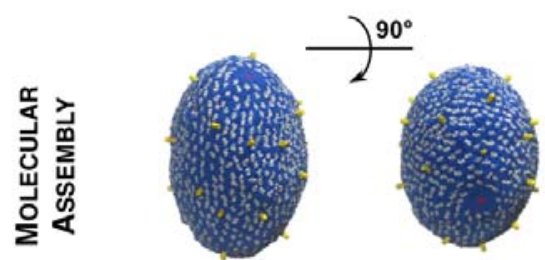

Model M1



M1, T $=4 \mu \mathrm{s}$

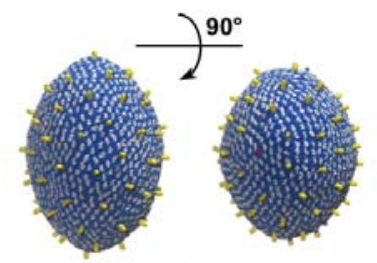

Model M2



$\mathrm{M} 2, \mathrm{~T}=4 \mu \mathrm{s}$

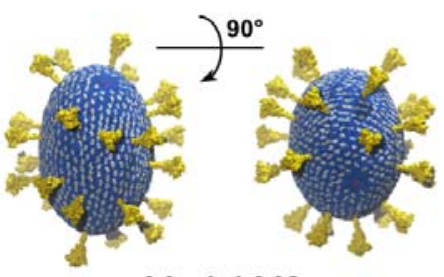

Model M3

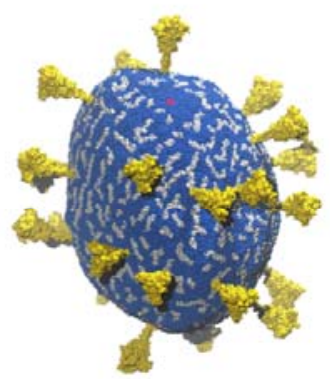

$\mathrm{M3}, \mathrm{T}=1 \mu \mathrm{s}$
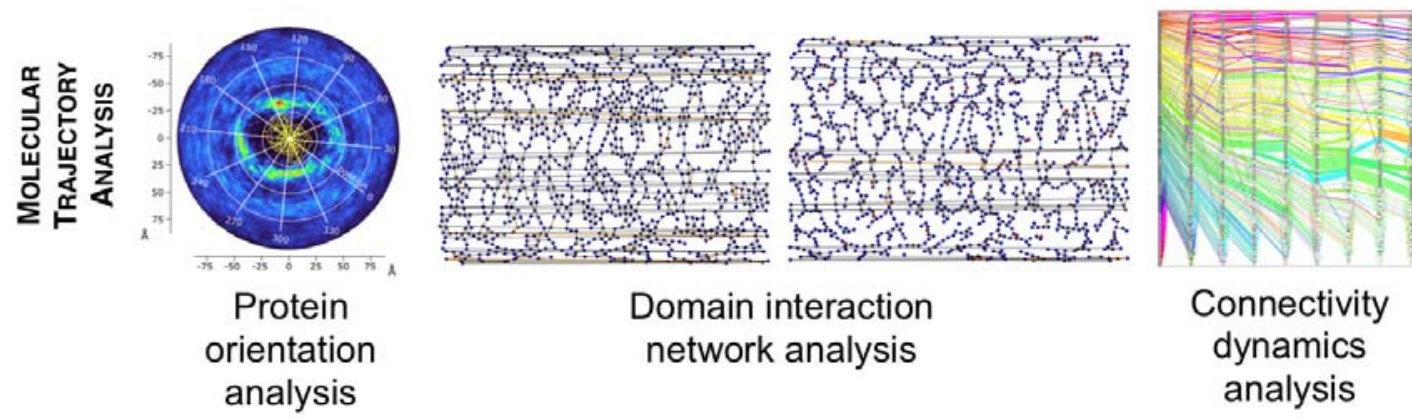

Figure 1. Key stages of integrative computational modeling pipeline: First, the structural characterization of S, M, and E proteins in both, monomeric and homooligomeric, forms was carried out. Second, fingerprinting analysis was performed 
to determine the enrichment of specific lipids due to interactions with the proteins. Third, assembly of an initial structural configuration of the envelope was constructed by integrating data from multiple sources. Fourth, the molecular dynamics simulation of several configurations was performed. Last, structural and network analysis of the obtained structural trajectories was done to reveal characteristic patterns of the structural proteins in the envelope assembly. 



Figure 2. Structural characterization of SARS-CoV-2 viral envelope and its components: A. An envelope model obtained from configuration C1 (2 E pentamers, 25 $\mathrm{S}$ trimers, $1003 \mathrm{M}$ dimers) and including full-length structures of $\mathrm{S}$ trimers after $1 \mu \mathrm{s}$ simulation run. Lipid molecules are depicted in sapphire blue, E pentamers in ruby red, $\mathrm{M}$ dimers in silver, and $\mathrm{S}$ trimers in gold. Principal diameters have values of $81.3 \mathrm{~nm}$, $97.8 \mathrm{~nm}$, and $103.1 \mathrm{~nm}$. Height of the outer part of S protein is $25 \mathrm{~nm}$. Surface of the envelope displays "filament" patterns formed by transmembrane domains of M dimers, while the internal part of the envelope shows tight packing of M dimers' endodomains assemblies; B. Envelope model from configuration C1 using truncated S trimer structures at the start of the simulations (top) and after $4 \mu$ s (bottom); C. Structural proteins S, E, and $\mathrm{M}$ representing the main structural building blocks of SARS-CoV-2 envelop in their physiological oligomeric states: $\mathrm{S}$ trimer, E pentamer, and $\mathrm{M}$ dimer. The grey dashed lines correspond to the membrane boundaries. The structures are shown in different scales. D. Change of the viral shape during the simulation defined through the principal gyration radii. The two largest principal radii converge to the value $\sim 28 \mathrm{~nm}$ while the third one converges to $\sim 24 \mathrm{~nm}$. The actual diameters of the model after $4 \mu$ s simulation were $103.1 \mathrm{~nm}, 97.8 \mathrm{~nm}$, and $81.3 \mathrm{~nm}$, respectively. 

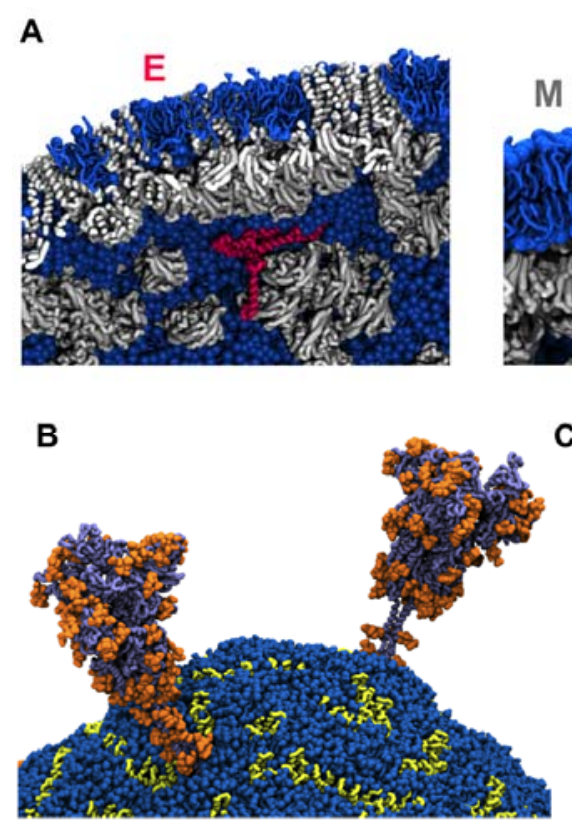



intra-cellular
C



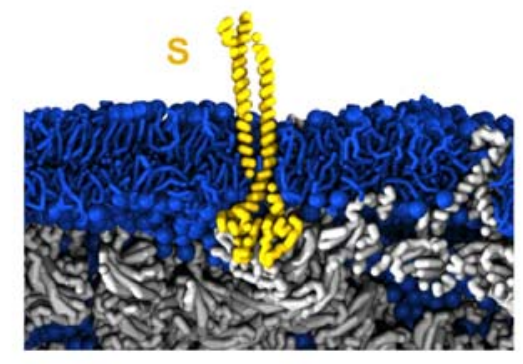

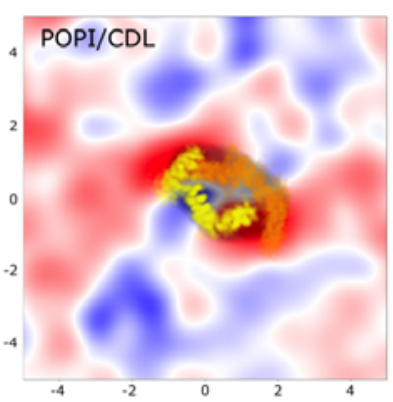

Figure 3. Protein-lipid interactions in the envelop model: A. Local view of the molecular interactions between lipids (sapphire blue) and structural proteins: E pentamer (ruby red), M dimer (silver), and S trimer (gold). Unlike E pentamer that remained fully surrounded by the lipid molecules, every $S$ trimer became supported through molecular interaction at least by one $\mathrm{M}$ dimer at the end of the simulation. Similarly, most $\mathrm{M}$ dimers were found interacting with other $\mathrm{M}$ dimers. B. Two glycosylated S trimers (dark grey) shown together with their glycans (orange) after $1 \mu$ s simulation run. C. Lipid fingerprinting analysis showed a slight enrichment of the negative lipids around $\mathrm{M}$ dimers. 
A

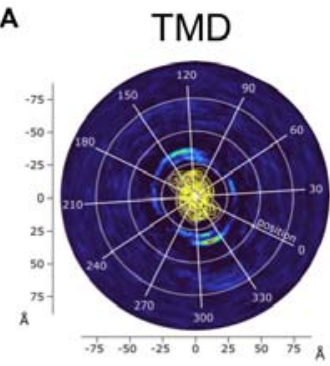

$\mathrm{C}_{-75}$



E

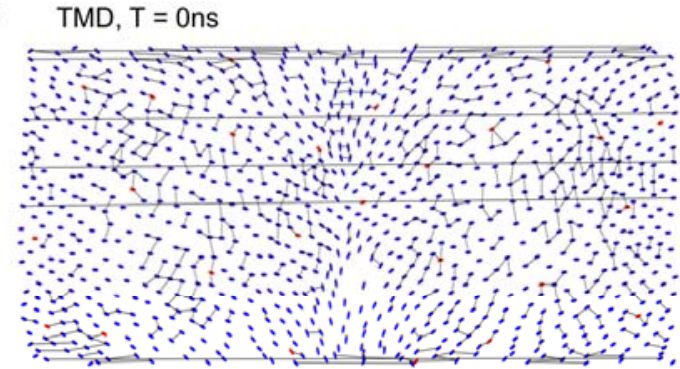

$E D, T=$ Ons

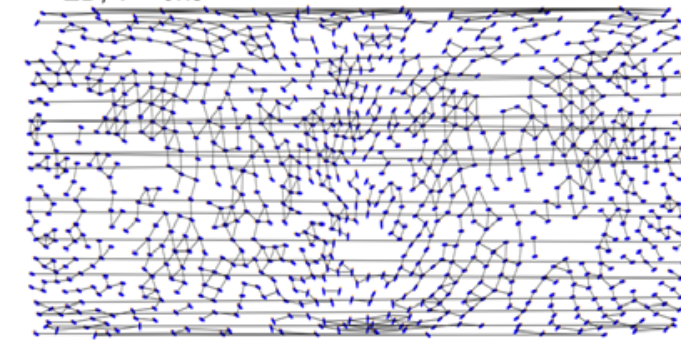

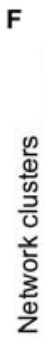
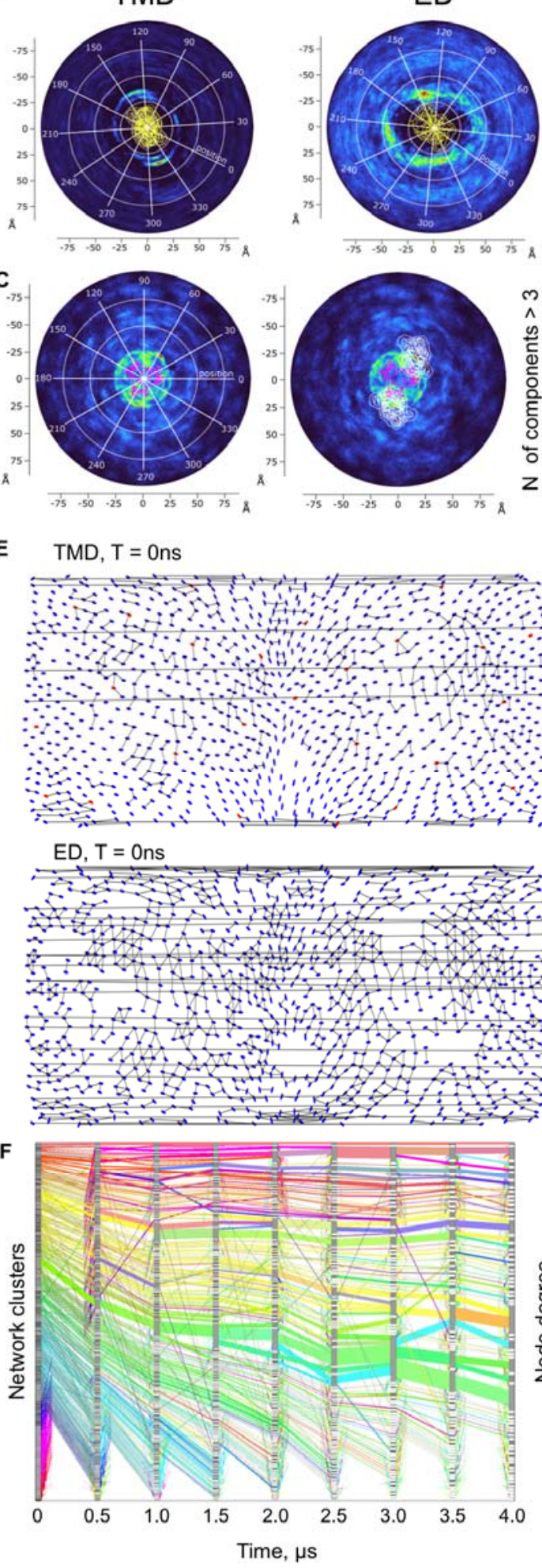

B

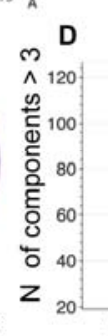

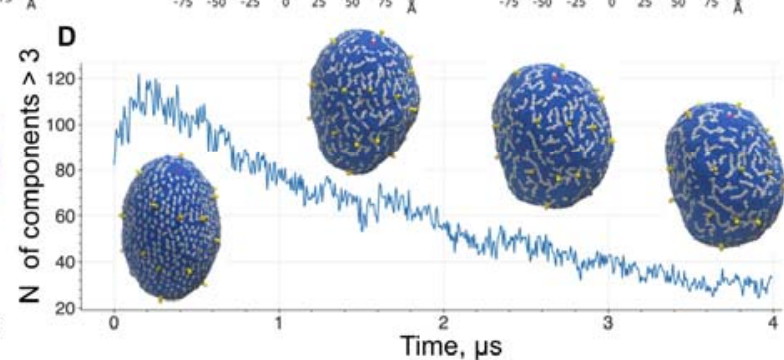

TMD, $\mathrm{T}=4,000 \mathrm{~ns}$



$\mathrm{ED}, \mathrm{T}=4,000 \mathrm{~ns}$

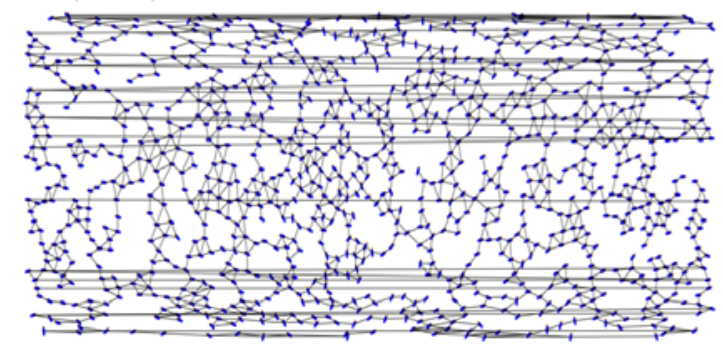

G



Figure 4. Structural and network analysis of the envelope assembly. A. Orientation preference of during molecular interactions for the transmembrane domains (TMD) and endodomains (ED), two main components of $\mathrm{M}$ protein in their dimeric state. TMD components display two preferred locations at $135^{\circ}$ and $315^{\circ}$, ED components display 
four preferable locations: $135^{\circ}, 315^{\circ}, 225^{\circ}$ and $75^{\circ}$; B. Super position of averaged CryoEM images previously obtained from SARS-CoV envelope with $\mathrm{M}$ dimer models obtained separately for TMDs and EDs and arranged according to the preferred interaction positions from panel A, demonstrating a near-perfect correspondence between our model and the Cryo-EM images. C. Orientation analysis of the contacts between $\mathrm{S}$ trimers and $\mathrm{M}$ dimers. Shown are positions of the $\mathrm{S}$ trimer (pink), the density of positions of $\mathrm{M}$ dimers (left panel) and the same density together with two $\mathrm{M}$ dimers (white) positioned around two distinct high-density locations at $60^{\circ}$ and $260^{\circ}$ (right panel). D. The number of connected components that have at least three nodes dynamically change during the simulation; included into the same plot are envelope models at $\mathrm{t}=0 \mu \mathrm{s}, 1.5 \mu \mathrm{s}$, $2.5 \mu \mathrm{s}$, and $4 \mu \mathrm{s}$ from configuration $\mathrm{C} 1$. E. Mercator projection of physical domaindomain interaction network established for molecular interactions of $\mathrm{M}$ dimers (blue ellipses) with each other and with $\mathrm{S}$ trimer (red ellipses) for the models from configuration $\mathrm{C} 1$ (2 E pentamers, $25 \mathrm{~S}$ trimers, $1003 \mathrm{M}$ dimers). M dimer's TMDs and EDs are arranged into separate domain-domain interaction networks. The orientation of ellipses corresponds to the orientation of the corresponding oligomers. An ellipse with the major axis positioned horizontally corresponding to the canonical orientations of the oligomers, as defined in panels $\mathrm{A}$ and $\mathrm{C}$, for $\mathrm{M}$ dimer and $\mathrm{S}$ trimer, respectively. Connectivity increases after $4 \mu$ s of simulations for both TMD and ED components; $\mathbf{F}$. Sankey diagram showing clustering dynamics of the TMD components over the time for the model in configuration $\mathrm{C} 1$. Each of the displayed "flows" contains at least 25 network nodes. One can see a drastic increase in the cluster size for a small number of connected components as the simulation progresses; G. Average node degree dynamics during the simulation for TMDs (top) and EDs (bottom). Yellow bands indicate values for the $2^{\text {nd }}$ and $3^{\text {rd }}$ quantiles, black lines denote the minimum and maximum values. There is a clear trend for the increase of the node degree value for TMD components; the value plateaus during the last microsecond $(3-4 \mu \mathrm{s})$ around the value of 2.3. EDs' node degrees have a much smaller spread and tend to converge to the value of 3.2. 
Table 1. Number of Martini3 particles per category in models. Show are four simulations two for a model in configuration C2 (3 E pentamers, $71 \mathrm{~S}$ trimers, and 1080 $\mathrm{M}$ dimers) and two for configuration C1 (2 E pentamers, $25 \mathrm{~S}$ trimers, $1003 \mathrm{M}$ dimers) with truncated (short) and full S trimers. A CG water particle corresponds to 4 real water molecules.

\begin{tabular}{|c|c|c|c|c|c|}
\hline Composition & & $\mathrm{C} 2,4 \mu \mathrm{s}$ & $\mathrm{C} 2,1 \mu \mathrm{s}$ & $\begin{array}{c}\text { C1 (short S), } \\
4 \mu \mathrm{s}\end{array}$ & $\begin{array}{c}\text { C1 (full S), } \\
1 \mu \mathrm{s}\end{array}$ \\
\hline \multirow{4}{*}{ Proteins } & $S$ & 71 & 71 & 25 & 25 \\
\hline & $\mathrm{M}$ & 3 & 3 & 2 & 2 \\
\hline & $E$ & 1,080 & 1,080 & 1,003 & 1,003 \\
\hline & $\begin{array}{c}\text { Total } \\
\text { particles }\end{array}$ & $1,056,909$ & $1,056,909$ & 968,373 & $1,237,398$ \\
\hline \multirow{7}{*}{ Lipids } & POPC & 39,755 & 24,187 & 34,923 & 34,923 \\
\hline & POPE & 13,191 & 8,061 & 11,837 & 11,837 \\
\hline & POPI & 7,195 & 4,397 & 5,918 & 5,918 \\
\hline & POPS & 0 & 0 & 1,183 & 1,183 \\
\hline & CHOL & 0 & 0 & 2,663 & 2,663 \\
\hline & CDL2 & 0 & 0 & 2,663 & 2,663 \\
\hline & $\begin{array}{c}\text { Total } \\
\text { particles }\end{array}$ & 736,082 & 448,534 & 751,373 & 751,373 \\
\hline \multirow{4}{*}{ Solvent } & $\mathrm{Na}$ & 50,000 & 175,764 & 175,565 & 409,000 \\
\hline & $\mathrm{Cl}$ & 66,169 & 194,719 & 184,999 & 517,855 \\
\hline & Water & $14,668,645$ & $21,820,675$ & $13,045,399$ & $34,236,835$ \\
\hline & $\begin{array}{c}\text { Total } \\
\text { particles }\end{array}$ & $14,784,814$ & $22,191,158$ & $13,220,964$ & $35,163,690$ \\
\hline
\end{tabular}




\section{References}

1 De Wit, E., Van Doremalen, N., Falzarano, D. \& Munster, V. J. SARS and MERS: recent insights into emerging coronaviruses. Nature Reviews Microbiology 14, 523-534 (2016).

2 Li, R. et al. Substantial undocumented infection facilitates the rapid dissemination of novel coronavirus (SARS-CoV-2). Science 368, 489-493 (2020).

3 Zhou, P. et al. A pneumonia outbreak associated with a new coronavirus of probable bat origin. nature 579, 270-273 (2020).

4 Kim, J. et al. Advanced bioinformatics rapidly identifies existing therapeutics for patients with coronavirus disease-2019 (COVID-19). Journal of translational medicine 18, 1-9 (2020).

5 Mandala, V. S. et al. Structure and drug binding of the SARS-CoV-2 envelope protein transmembrane domain in lipid bilayers. Nature structural \& molecular biology 27, 1202-1208 (2020).

6 Yao, H. et al. Molecular architecture of the SARS-CoV-2 virus. Cell 183, 730-738. e713 (2020).

7 Liu, C. et al. The architecture of inactivated SARS-CoV-2 with postfusion spikes revealed by cryo-EM and cryo-ET. Structure 28, 1218-1224. e1214 (2020).

8 Neuman, B. W. et al. A structural analysis of M protein in coronavirus assembly and morphology. Journal of structural biology 174, 11-22 (2011).

9 Rahman, M. S. et al. Mutational insights into the envelope protein of SARS-CoV-2. Gene reports 22, 100997 (2021).

10 Godet, M., L'Haridon, R., Vautherot, J.-F. \& Laude, H. TGEV corona virus ORF4 encodes a membrane protein that is incorporated into virions. Virology 188, 666-675 (1992).

11 DeDiego, M. L. et al. A severe acute respiratory syndrome coronavirus that lacks the E gene is attenuated in vitro and in vivo. Journal of virology 81, 1701-1713 (2007).

12 Huang, Y., Yang, Z.-y., Kong, W.-p. \& Nabel, G. J. Generation of synthetic severe acute respiratory syndrome coronavirus pseudoparticles: implications for assembly and vaccine production. Journal of virology 78, 12557-12565 (2004).

13 Kuo, L., Hurst-Hess, K. R., Koetzner, C. A. \& Masters, P. S. Analyses of coronavirus assembly interactions with interspecies membrane and nucleocapsid protein chimeras. Journal of virology 90, 4357-4368 (2016).

14 Siu, Y. et al. The M, E, and N structural proteins of the severe acute respiratory syndrome coronavirus are required for efficient assembly, trafficking, and release of virus-like particles. Journal of virology 82, 11318-11330 (2008).

Neuman, B. W. et al. in The Nidoviruses 181-185 (Springer, 2006).

Jefferys, E. E. \& Sansom, M. S. in Physical Virology 201-233 (Springer, 2019).

Woo, H. et al. Developing a fully glycosylated full-length SARS-CoV-2 spike protein model in a viral membrane. The Journal of Physical Chemistry B 124, 7128-7137 (2020).

18 Srinivasan, S. et al. Structural genomics of SARS-CoV-2 indicates evolutionary conserved functional regions of viral proteins. Viruses 12, 360 (2020).

19 Heo, L. \& Feig, M. Modeling of severe acute respiratory syndrome coronavirus 2 (SARS-CoV-2) proteins by machine learning and physics-based refinement. BioRxiv (2020).

20 Pezeshkian, W., König, M., Marrink, S. J. \& Ipsen, J. H. A multi-scale approach to membrane remodeling processes. Frontiers in molecular biosciences 6, 59 (2019).

21 Pezeshkian, W. \& Ipsen, J. H. Fluctuations and conformational stability of a membrane patch with curvature inducing inclusions. Soft matter 15, 9974-9981 (2019).

Pezeshkian, W., König, M., Wassenaar, T. A. \& Marrink, S. J. Backmapping triangulated surfaces to coarse-grained membrane models. Nature communications 11, 1-9 (2020).

Marrink, S. J., Risselada, H. J., Yefimov, S., Tieleman, D. P. \& De Vries, A. H. The MARTINI force field: coarse grained model for biomolecular simulations. The journal of physical chemistry $B$ 111, 7812-7824 (2007).

Wassenaar, T. A., Pluhackova, K., Bo $\square$ ckmann, R. A., Marrink, S. J. \& Tieleman, D. P. Going backward: a flexible geometric approach to reverse transformation from coarse grained to atomistic models. Journal of chemical theory and computation 10, 676-690 (2014).

Kern, D. M. et al. Cryo-EM structure of SARS-CoV-2 ORF3a in lipid nanodiscs. Nature Structural \& Molecular Biology, 1-10 (2021). 

coronavirus revealed by electron cryomicroscopy. Journal of virology 80, 7918-7928 (2006).

27 Klein, S. et al. SARS-CoV-2 structure and replication characterized by in situ cryo-electron tomography. Nature communications 11, 1-10 (2020).

28 Wassenaar, T. A. et al. High-throughput simulations of dimer and trimer assembly of membrane proteins. The DAFT approach. Journal of chemical theory and computation 11, 2278-2291 (2015).

29 Bárcena, M. et al. Cryo-electron tomography of mouse hepatitis virus: Insights into the structure of the coronavirion. Proceedings of the National Academy of Sciences 106, 582-587 (2009).

30 Liu, C. et al. Viral architecture of SARS-CoV-2 with post-fusion spike revealed by Cryo-EM. BioRxiv (2020).

31 Yu, A. et al. A multiscale coarse-grained model of the SARS-CoV-2 virion. Biophysical journal 120, 1097-1104 (2021).

32 Galindo, I. et al. Antiviral drugs targeting endosomal membrane proteins inhibit distant animal and human pathogenic viruses. Antiviral research 186, 104990 (2021).

33 Dodd, R. B., Wilkinson, T. \& Schofield, D. J. Therapeutic monoclonal antibodies to complex membrane protein targets: Antigen generation and antibody discovery strategies. BioDrugs 32, 339-355 (2018).

34 Veneziano, R. et al. Role of nanoscale antigen organization on B-cell activation probed using DNA origami. Nature nanotechnology 15, 716-723 (2020). 


\section{STAR METHODS}

1 . Modeling and structural refinement of M-dimer

Unlike for proteins $\mathrm{S}$ and $\mathrm{E}$, no experimental structures have been solved for SARS-CoV-2 M protein or any of its homologs, neither as a monomer nor as a dimer, which is its physiological conformation in the envelope. Thus, a comparative modeling approach cannot be applied, and a novel integrative approach was introduced that utilized geometric constraints of the M-dimer in the envelope derived from the low-resolution CryoEM images of the envelopes of the closely related coronaviruses, SARS-CoV and MHV, as well as a high-resolution CryoEM structure of a dimer for another SARS-CoV-2 protein sharing substantial structural similarity (see Supp. Fig. $\mathrm{S} 2, \mathrm{~S} 3)$.

The approach includes six steps. First, a model of $\mathrm{M}$ monomer was obtained using de novo modeling methods AlphaFold and I-TASSER ${ }^{1-3}$. The top 10 models were then selected and the models of $\mathrm{M}$ homodimers were obtained using two symmetric docking approaches, SymDock and Galaxy ${ }^{4-6}$. Next, a set of geometric constraints was applied to an ensemble of the 100 topscoring homodimer models. The geometric constrains include the dimer axial dimensions and the shape of the part of the packaged dimer located on the envelope surface, the orientation of the monomers, and the dimensions of the transmembrane domain (TMD) of the packaged M-dimer defined by the envelope membrane's thickness. Specifically, from the previous analysis of SARS$\mathrm{CoV}$ envelope ${ }^{7,8}$, it follows that the M-dimers form a lattice with a unit cell made of four Mdimers, representing a parallelogram with angles of $100^{\circ}$ and $80^{\circ}$, and sides of $6.0 \mathrm{~nm}$ and $7.5 \mathrm{~nm}$ measured between the dimer centers (Fig. 1). Furthermore, the dimers are expected to have Ntermini of both monomers located on the exterior surface of the virion's envelope, and the thickness of the envelope's membrane is set to be equal to $4 \mathrm{~nm}^{9,10}$.

The analysis of the three top scoring M-dimers that satisfy all above geometric constraints provided us with an interesting finding: all three dimer models share striking structural similarity with ORF3 protein of SARS-CoV-2 (Supp. Fig. S3), whose CryoEM structure was recently solved ${ }^{11}$. In particular, we found that the de novo modeled structure of the endodomain of $\mathrm{M}$ and the experimentally obtained structure of C-terminal domain of ORF3 are structurally similar, while the transmembrane domain structure of $\mathrm{M}$ and $\mathrm{N}$-terminal domain of ORF3 shared the same secondary structure elements (four helices) of the same lengths while the mutual positions of the helices were somewhat different. Therefore, we hypothesized that M-dimers and ORF3a are structurally similar, and the model of the M-dimer could be further refined using the structural information from ORF3a (Fig. 1B). To use the structural information from ORF3a, we first created a "fragmented' structural template by individually structurally aligning the four helices and endodomain of $\mathrm{M}$ dimer with the corresponding helices and endodomain in the ORF3a template structure (PDB ID: 6XDC). Then, we obtained a preliminary comparative model using the newly created fragmented structural template of M-dimer. The obtained M-dimer model was refined using a protocol similar to the one used to obtain a full-length model S-trimer (Fig. S4) ${ }^{12}$. First, the linker regions of the two TMDs in the obtained comparative model of M-dimer were refined by energy minimization, followed by refinement of the whole TMDs using Molecular Modeling Toolkit ${ }^{13}$. Next, the overall M-dimer structure was minimized using the CHARMM36 force field in GROMACS ${ }^{14}$. We then placed the M-dimer model into the experimental EM density map of the ORF3a dimer (EMD-22139 ${ }^{11}$ ) using Phenix ${ }^{15}$ and relied on the EM map to further refine the structure in ISOLDE ${ }^{16}$, a package for UCSF Chimera X molecular visualization program ${ }^{17}$. ISOLDE uses OpenMM-based interactive molecular dynamics flexible fitting ${ }^{18}$ using AMBER force field ${ }^{19}$ and allows for the real-time assessment and validation of the geometric clashing problems. Each residue of the M-dimer model (1-946) was then inspected and remodeled to maximize its fit into the density map. We considered both a deposited electron-microscopy 
map and a smoothed version with a B-factor of $100 \AA^{2}$ as proposed in ${ }^{12}$, but in our case there was no significant difference between these two versions of the refinement protocol.

\section{Overall stoichiometry of the envelope}

The molecular composition of the SARS-CoV-2 envelope and the stoichiometries of the structural proteins have been determined based on the current information obtained from CryoEM experiments, by analyzing the stoichiometries of other betacoronaviruses, and through arranging the three structural proteins into a geometrically constrained shape of the envelope. Comparable numbers of $S$ trimers have been previously reported across other beta coronaviruses. An early model of BECV reported up to $110 \mathrm{~S}$ trimers ${ }^{20}$, while another early model of TGEV provided a rough estimate of 100-200 S trimers ${ }^{21}$. SARS-CoV has been determined to contain $\sim 90$ copies of $\mathrm{S}$ trimers ${ }^{8}$ per particle. Interestingly, the number of S trimers in a SARS-CoV-2 recently obtained from the cryoelectron tomography experiments was substantially less, namely $26 \pm 15 \mathrm{~S}$ trimers ${ }^{22}$. A similar estimate of $24 \pm 9 \mathrm{~S}$ trimers was reported independently ${ }^{23}$. The numbers of $\mathrm{M}$ molecules per virion are also consistent across studied betacoronaviruses. For SARS-CoV, MHV and $\mathrm{FCoV}$, it was estimated at $1,100 \mathrm{M}$ dimers on average per particle ${ }^{8}$. Finally, E pentamers has been reported to localize in the envelope membrane in minute amounts ${ }^{8,24}$. In TGEV, 15-30 molecules of E protein, which corresponds to 3-6 pentamers was predicted ${ }^{21}$. Similar numbers of $\sim 20$ copies of E molecules, which correspond to four pentamers have been reported for MHV ${ }^{25}$.

In our two main models, M1 and M3, we considered a molecular composition C1 that included $1003 \mathrm{M}$ dimers (2,006 monomers), $25 \mathrm{~S}$ trimers ( 75 monomers), and $2 \mathrm{E}$ pentamers (10 monomers). M1 had truncated S trimers (for more details see the next section), while M3 had the full-length spikes. The number of E-pentamers was slightly reduced from the estimates for other coronaviruses in order to maintain a $\mathrm{E}: \mathrm{S}$ molar ratio close to the one reported for TGEV ${ }^{21}$. In addition, to explore if the differences between the structural proteins of SARS-CoV and SARS$\mathrm{CoV}-2$ do not allow to maintain an envelope structure with a higher number of spike proteins for the SARS-CoV-2 virion, we have also simulated another model, M2, with a composition, C2, similar to that one estimated or SARS-CoV ${ }^{8}$ that included $1,080 \mathrm{M}$ dimers, $71 \mathrm{~S}$ trimers, and $3 \mathrm{E}$ pentamers.

\section{Martini3 protein models}

The coarse-grained protein structures and input parameters were created from the atomistic reference structures of oligomers using martinize $2{ }^{26}$, following Martini3 guidelines for creating protein input parameters ${ }^{27}$. The parameters for $\mathrm{M}$ protein were based upon our own refined atomistic structure, whereas parameters for $\mathrm{E}$ and $\mathrm{S}$ proteins were derived from the previously published models ${ }^{12,28}$. An elastic network was used to keep the secondary structure of the proteins fixed as required in CG simulations with Martini3. For E protein, we created a custom elastic network, where the transmembrane domains of all 5 monomers were connected to keep the channel's stability. In addition, the elastic network of the intracellular domains were applied only within each monomer to allow for flexibility. For S protein, two CG models were created: (1) a model that included copies of the whole $S$ protein together with glycosylation, and (2) a model that included a truncated structure of S consisting of HR2, TM, and CT domains in close proximity to the envelope (resid 1172-1273). Coordinates and parameters for the glycosylation of the full S protein were generated in a separate step using the polyply program ${ }^{29}$ and a Martini3 beta sugar model with adopted parameters for the final Martini3 version. Composition of the glycans and attachment sites were considered the same as in the previously published work ${ }^{28}$, except for the O-glycan, which was omitted due to missing parameters. No elastic network was applied to the glycans.

\section{Lipid fingerprinting analysis}


While the lipid composition of the SARS-CoV-2 envelope has not been determined, it has been approximated as being close to the composition of the endoplasmic reticulum ${ }^{19,30}$. To analyze if any of the proteins have specific lipid interactions or favored lipid environments, the so-called lipid fingerprint was determined ${ }^{31,32}$. Specifically, we ran three systems consisting of $4 \mathrm{M}$ proteins, $4 \mathrm{M}$-proteins and 1 truncated S-protein, and $4 \mathrm{M}$-proteins and 1 E-protein in a flat lipid bilayer. The composition of the bilayer was POPC (0.55), POPE (0.25), POPI (0.10), POPS (0.02), cholesterol (0.06), and cardiolipin 2 (0.02), which approximated the composition found in the ER ${ }^{30}$. To account for the specific lipid binding in E pentamer, it was placed with its immediate lipid shell as obtained from a separate free simulation. Each of the systems was run for $4 \mu \mathrm{s}$, following the default simulation setup for Martini membranes ${ }^{33}$. Then, the depletion enrichment index and a 2D density map was computed by analyzing the lipid compositions as a function of distance to the protein over time.

\section{Building initial CG structure of the full envelope}

To build the initial CG structure of the envelope, we used a top-down protocol. First, by performing two types of Monte Carlo moves (vertex move and link flip ${ }^{34}$ ) on a spherical triangulated surface (TS) containing 1,030 and 1,154 vertices for models M1/M2 and M3, respectively, we drove the system to reshape to an ellipsoidal TS with aspect ratio of 0.67:0.89:1 to match an average shape reported in experimental measurements ${ }^{23}$. Next, we assigned the location of each vertex of the TS to a homo-oligomeric complex (M dimer, $\mathrm{S}$ trimer, or $\mathrm{E}$ pentamer). The vertices are assigned so that the spatial distribution of complexes is uniform. To find a proper orientation of the oligomeric complexes, we ran a Monte Carlo simulation on the orientation of these complexes using a dynamic triangulated surface (DTS) simulation method ${ }^{34}$ without updating the TS shape. To do so, we assumed that each homo-oligomer interacted only with the neighboring protein complexes by a potential defined as:

$$
E=-\varepsilon_{i j}(1+\cos N \theta)
$$

where $\varepsilon_{i j}$ is the interaction strength, $N$ is the least common multiple of the degree of the $i, j$-th complex symmetry in the plane of the membrane and $\theta$ is the angle between the inclusion vector (a vector representing the in-plane orientation of a homo-oligomer) residing on vertex $i$ and the inclusion vector residing on vertex $j$ after parallel transport to vertex $i^{35}$.

The final DTS output was then converted to a Martini model using TS2CG software ${ }^{36}$. Before any solvation of the CG structure, we first performed 100 steps of energy minimization using soft-core potential ${ }^{37}$, followed by a normal energy minimization for $10 \mathrm{k}$ steps and $1 \mathrm{k}$ steps molecular dynamics run to relax the chains lipid. Then, we solvated the system and added Na+ and $\mathrm{Cl}$ - to neutralize the system, with an overall ion concentration of $0.9 \%$. Finally, another energy minimization round is implemented followed by a short equilibration MD simulation run in NVT ensemble and a longer run in NPT ensemble using Berendsen barostat. The resulting arrangement is approaching a limit of physically possible fit in terms of the protein density with respect to the virion surface.

All CG-MD simulations were performed using the GROMACS package (versions 2019) and the Martini 3 force field. A time step of 20 fs was used, though for certain steps it was necessary to perform runs with shorter time steps (see 9-step protocol below). Both van der Waals (cutoff) and Coulomb interactions (reaction-field) were set to zero at $1.1 \mathrm{~nm}$ using the Verlet cutoff scheme, following recommended values for Martini-based simulations ${ }^{38}$. Coulomb interactions were screened by a relative permittivity constant of 15 . The systems were equilibrated first in an NVT, followed by NPT ensembles using the Berendsen barostat and the temperature was kept constant at $310 \mathrm{~K}$. The final production run was performed in the NPT ensemble, using the Parrinello- 
Rahman barostat with reference pressure of 1 bar and compressibility of $3 \times 10-4$ bar-1, respectively.

The lipid composition of the viral envelope was taken to be the same as the one used in the lipid fingerprint simulations, since no significant lipid preferences were found for the $\mathrm{M}$ protein during the lipid fingerprint analysis. Similar to the lipid fingerprinting analysis, to account for the specific lipid binding in the two E pentamers, each E pentamer was placed with its immediate lipid shell. This resulted in the final lipid bilayer composition of 39,755 POPC, 13,191 POPE, and 7,195 POPI lipids for M3 model and an expanded set of 34,923 POPC, 11,837 POPE, 5,918 POPI, 1,183 POPS, 2,663 CHOL, 2,663 CDL2 for the final models of M1/M2.

\section{Nine-step protocol with parameter settings:}

(1) Energy minimization with softcore potentials: 100 steps, $0.02 \mathrm{ps}$ time step, flexible water, steep integrator, V-rescale temperature coupling, 310K temperature, no pressure coupling.

(2) Energy minimization with positional restraints: 10,000 steps, 0.02ps time step, flexible water, steep integrator, no temperature and pressure coupling.

(3) Equilibration with positional restraints: 10,000 steps, $0.001 \mathrm{ps}$ time step, rigid water, sd integrator, V-rescale temperature coupling, $310 \mathrm{~K}$ temperature, no pressure coupling.

(4) Solvation with 1.5 angstrom distance to the protein in lipids and $0.9 \%$ salt ions content.

(5) Equilibration with positional restraints: 100,000 steps, other parameters are identical to 2)

(6) NVT equilibration with positional restraints: 500,000 steps, 0.002ps time step, rigid water, sd integrator, rigid water, V-rescale temperature coupling, $310 \mathrm{~K}$ temperature, no pressure coupling.

(7) Short run with positional restraints: 750,000 steps, 0.02ps time step, rigid water, md integrator, V-rescale temperature coupling, $310 \mathrm{~K}$ temperature, Berendsen barostat, isotropic pressure.

(8) NPT equilibration (no restraints): 100,000 steps, $0.01 \mathrm{ps}$ time step, rigid water, sd integrator, V-rescale temperature coupling, $310 \mathrm{~K}$ temperature, Berendsen barostat, isotropic pressure.

(9) Production run: 50,000,000 steps, 0.02ps time step, md integrator, Berendsen/ParinelloRahman barostat (first and second run correspondingly), isotropic pressure.

Production runs for the systems were performed on the TACC Frontera supercomputer on nodes equipped with Intel Xeon Platinum 8280 with 56 AVX_512 logical cores per node. Two models were simulated here: a full-spike $1 \mu$ s simulation that took $\sim 208,000$ and several $4 \mu$ s spike conformations simulations each taking $\sim 925,000 \mathrm{CPU} /$ hours on average.

\section{Analysis of macromolecular spatial organization}

Interactions between proteins were investigated using a computational method for analysis of relative orientations ${ }^{39}$. In a first pass, protein representations were reduced to their center of mass positions and orientations of pre-specified internal coordinate frames. The latter were determined for the M protein's TMD and endodomain separately in their dimeric forms, S-trimer, and Epentamer by aligning the axis of symmetry with the $z$ axis and aligning the vector from the center of mass of the multimer to the center of mass of the first monomer with the $x$ axis. The orientation for a protein complex is then obtained by a transformation required to align the reference orientation to the actual orientation, which is stored as the translation vector and the rotation matrix. This greatly reduces the number of degrees of freedom, allowing for more efficient follow-up analyses. 
In the second stage of the analysis, all pairs of protein complexes within a specific distance (10 $\mathrm{nm}$ ) were collected, and for each pair the orientations were expressed as relative orientations by aligning one partner with the Cartesian axes, i.e., by subtracting the center of mass and multiplying by the transpose of the rotation matrix. These relative orientations were subsequently expressed as a distance and five angles, adapted from the previous work ${ }^{39}$. For each protein, this yields an angle $\alpha$, which denotes the angle of the center of mass of the partner with the XY plane, and and angle $\beta$, which denotes the position of binding in the XY plane. The fifth angle captures the tilt, but is not investigated further in this work.

For all pairs of proteins within a given distance, their parameters were collected, and density plots were made to show which combinations of parameters were characteristic for each interaction. These characteristic orientations were used for assessment of the higher order structures. In addition, these orientations were combined with per-protein XY-plane particle densities to compare with the related experimental data obtained from the cryoelectron microscopy (EM) images of SARS-CoV ${ }^{8}$.

\section{Shape analysis}

To assess the shape of the SARS-CoV-2 envelope the eigenvalues of the gyration tensor as well as two shape descriptors, $S$ and Delta, were computed following their analysis ${ }^{40}$. Delta descriptor measures the asphericity of a particle distribution with a lower bound of 0 , which indicates a perfect spherical distribution. $S$ descriptor, in contrast, can have negative values indicating a point distribution corresponding to an oblate spheroid and positive values indicating a distribution corresponding to a prolate spheroid. Furthermore, to measure convergence of the simulation we estimated the auto-correlation and equilibration times as previously proposed ${ }^{41,42}$. In addition to this global shape analysis, a more detailed view was determined following a procedure derived from an efficient method to calculate a molecular hull and contact bodies ${ }^{43}$, which consists of extruding a triangulated sphere to the surface of the virion, in this case marked by the M dimer TMD centers of mass. The extrusion of each vertex was determined from a kernel density estimate of TMD distances from the center in angular coordinates. This yields a smoothed triangulated surface through the membrane center, which allows visual inspection of the dynamics of the shape, and from which shape volumes and surface areas can be calculated easily.

\section{Protein network analysis}

To get further insights into the dynamic reordering of structural proteins that took place in the envelope, we conducted the connectivity analysis, constructing domain-level protein-protein interaction networks. This approach was a scaled-up version of the protein structure network (PSN) analysis, which was previously employed in the structural characterization of individual proteins ${ }^{44-47}$. Here, we defined a network node not as an individual amino acid residue, but as an entire protein domain. In this analysis, we differentiated only between the proteins' transmembrane domains (TMD) and endodomains (ED), which resulted in two separate physical contact networks: one within the lipid membrane and one on the inside of the virion.

We processed the reduced trajectory files (see Section 6 in Materials and Methods) that preserve the center of mass and orientation for each protein, substituting each entry with the canonical pdb model used in this study and conducting contact analysis with a cutoff distance of $0.6 \mathrm{~nm}$, a frequently used threshold for the coarse-grained structures ${ }^{48-50}$. This procedure is performed separately on TMDs and EDs of the structural proteins. As a result, two sets of temporal dynamic networks for each of the truncated spike models, M1 and M3, were obtained. Each temporal dynamic network was a series of domain-domain interaction network snapshots, taken every $1 \mathrm{~ns}$, from $0 \mu \mathrm{s}$ to $4 \mu \mathrm{s}$. Several key network properties were calculated for each network snapshot: 
average degree, number of connected components, average diameter, transitivity, and average degree connectivity for nodes with degrees 1,2 , and 3 .

The degree $d_{i}$ of a node $i$ is the number of edges incident to it. The average degree in a network of $N$ nodes is calculated according to the following formula: $d_{A}=\frac{1}{N} \sum_{i=1}^{N} d_{i}$. The number of connected components is the minimal number of subgraphs that have a path for any pair of vertices. The diameter of a graph $G$ is the maximum length of the shortest paths for each pair of vertices ${ }^{51}: \max _{i, j \in G} \delta(i, j)$, where $\delta(i, j)$ is a shortest path between vertices $i$ and $j$. Because our network was composed of multiple connected components, we calculated the diameter for each connected subgraph, and defined an average of the diameters: $D_{A}=\frac{1}{n} \sum_{s \in S} \max _{i, j \in S} \delta(i, j)$, where $S$ is a set of connected components and $n$ is its cardinality. The transitivity (also called the clustering coefficient) is the relative number of triangles (\#triangles) present in a given network compared to the number of all possible triangles (\#triads): $T=3 \frac{\# \text { triangles }}{\text { \#triads }}{ }^{52}$. Finally, the average $k$-degree connectivity is the average degree of the nearest neighbors for the nodes with the degree $k^{53}$. In this study, we computed the average degree connectivity for $k=1,2$, and 3 .

The number of connected components over time was fit with exponential and biexponential models:

$$
\begin{gathered}
\operatorname{Exp}(x)=c_{2} e^{c_{1} x}+c_{0} \\
\operatorname{BiExp}(x)=c_{4} e^{c_{3} x}+c_{2} e^{c_{1} x}+c_{0}
\end{gathered}
$$

The resulting models were compared using Akaike's information criterion (AIC) and Bayesian information criterion (BIC) ${ }^{54}$. Results were plotted using Python $3.9^{55}$ and Bokeh library ${ }^{56}$. A Sankey diagram of connected components was plotted in MATLAB ${ }^{57}$ using visualization module of PisCES algorithm ${ }^{58}$.

\section{Visualization of simulation trajectories}

Renderings of the virus envelope structure and molecular dynamics simulation were produced using VMD ${ }^{59}$ on TACC Frontera's large-memory nodes with 2.1 TB NVDIMM memory. A coordinate file containing the molecular structure was used as an input for VMD. Once loaded, each coarse grain bead was represented as a sphere with a radius three times its van der Waals radius. Solvent molecules were excluded from the visualization. To capture the whole dynamic simulation process, one frame per one nanosecond was extracted from the original trajectory using gmx trjconv ${ }^{37}$; the extracted XTC file containing the frames was then loaded into VMD. Lastly, a $t c l$ script was used to visualize each frame, followed by rendering the frames into TGA images using tachyon ${ }^{60}$.

Movie 1 was made by rendering each frame in the $4 \mu$ s trajectory of the envelope model M1 and rotating 2,000 steps around y-axis clockwise and 2,000 steps counterclockwise; each step was 0.9 degrees. Movie 2 was made with imagemagick montage ${ }^{61}$ by showing two identical $4 \mu \mathrm{s}$ trajectories, first one revealing the outside and the second one revealing the inside of the envelope's structural model M1. For Movie 3 representing a $1 \mu$ s trajectory of model M3, we used FFmpeg to convert images into a MP4 movie with $60 \mathrm{fps}$. Rendering a movie with 4,000 frames corresponding to $4 \mu$ s of simulation took $\sim 670 \mathrm{CPU}$-hours on TACC Frontera supercomputer.

To visualize the protein network (Movies 4 and 5), we first convert each node's 3D position into its $2 \mathrm{D}$ projection. We used the Mercator projection, which is a cylindrical projection that preserves local directions and shapes. Specifically, for each node, its $2 \mathrm{D}$ projection $(\mathrm{a}, \mathrm{b})$ is calculated by:

$$
r=\sqrt{x^{2}+y^{2}+z^{2}}
$$




$$
\begin{gathered}
b=y / r \\
\mathrm{a}=\tan ^{-1} x / z
\end{gathered}
$$

where $(\mathrm{x}, \mathrm{y}, \mathrm{z})$ is its normalized $3 \mathrm{D}$ position. The orientation of each protein domain is also converted in a similar manner. Then, we plot each node as a ellipse, where the its long axis follows the orientation and a orange tip suggests the direction, and colored spike proteins as red nodes and membrane proteins as blue nodes. The edges are represented as black lines where the new edges from the previous frame are highlighted as orange. We generated 1 frame per $10 \mathrm{~ns}$, which resulted in 400 frames corresponding to $4 \mu$ s of simulation.

1 Senior, A. W. et al. Improved protein structure prediction using potentials from deep learning. Nature 577, 706-710 (2020).

2 Yang, J. et al. The I-TASSER Suite: protein structure and function prediction. Nature methods $\mathbf{1 2}$, 7-8 (2015).

3 Zhang, Y. I-TASSER server for protein 3D structure prediction. BMC bioinformatics 9, 1-8 (2008).

4 André, I., Bradley, P., Wang, C. \& Baker, D. Prediction of the structure of symmetrical protein assemblies. Proceedings of the National Academy of Sciences 104, 17656-17661 (2007).

5 Shin, W.-H., Lee, G. R., Heo, L., Lee, H. \& Seok, C. Prediction of protein structure and interaction by GALAXY protein modeling programs. Bio Design 2, 1-11 (2014).

6 Ko, J., Park, H., Heo, L. \& Seok, C. GalaxyWEB server for protein structure prediction and refinement. Nucleic acids research 40, W294-W297 (2012).

7 Neuman, B. W. et al. in The Nidoviruses 181-185 (Springer, 2006).

8 Neuman, B. W. et al. A structural analysis of M protein in coronavirus assembly and morphology. Journal of structural biology 174, 11-22 (2011).

9 Fujiwara, H., Fujihara, M., Koyama, T. \& Ishiwata, T. in AIP Conference Proceedings. 724-726 (American Institute of Physics).

10 Klein, S. et al. SARS-CoV-2 structure and replication characterized by in situ cryo-electron tomography. Nature communications 11, 1-10 (2020).

11 Kern, D. M. et al. Cryo-EM structure of SARS-CoV-2 ORF3a in lipid nanodiscs. Nature Structural \& Molecular Biology, 1-10 (2021).

12 Woo, H. et al. Developing a fully glycosylated full-length SARS-CoV-2 spike protein model in a viral membrane. The Journal of Physical Chemistry B 124, 7128-7137 (2020).

13 Hinsen, K. The molecular modeling toolkit: a new approach to molecular simulations. Journal of Computational Chemistry 21, 79-85 (2000).

14 Huang, J. \& MacKerell Jr, A. D. CHARMM36 all $\square$ atom additive protein force field: Validation based on comparison to NMR data. Journal of computational chemistry 34, 2135-2145 (2013).

15 Liebschner, D. et al. Macromolecular structure determination using X-rays, neutrons and electrons: recent developments in Phenix. Acta Crystallographica Section D: Structural Biology 75, 861-877 (2019).

Croll, T. I. ISOLDE: a physically realistic environment for model building into low-resolution electron-density maps. Acta Crystallographica Section D: Structural Biology 74, 519-530 (2018).

17 Pettersen, E. F. et al. UCSF ChimeraX: Structure visualization for researchers, educators, and developers. Protein Science 30, 70-82 (2021).

18 Eastman, P. et al. OpenMM 7: Rapid development of high performance algorithms for molecular dynamics. PLoS computational biology 13, e1005659 (2017).

19 Case, D. A. et al. Amber 2020. (2020).

20 Roseto, A. et al. Bovine enteric coronavirus structure as studied by a freeze-drying technique. Journal of General Virology 63, 241-245 (1982).

21 Godet, M., L'Haridon, R., Vautherot, J.-F. \& Laude, H. TGEV corona virus ORF4 encodes a membrane protein that is incorporated into virions. Virology 188, 666-675 (1992).

Yao, H. et al. Molecular architecture of the SARS-CoV-2 virus. Cell 183, 730-738. e713 (2020).

$\mathrm{Ke}, \mathrm{Z}$. et al. Structures and distributions of SARS-CoV-2 spike proteins on intact virions. Nature 588, 498-502 (2020). 
Rahman, M. S. et al. Mutational insights into the envelope protein of SARS-CoV-2. Gene reports 22, 100997 (2021).

DeDiego, M. L. et al. A severe acute respiratory syndrome coronavirus that lacks the E gene is attenuated in vitro and in vivo. Journal of virology 81, 1701-1713 (2007). (Springer, 2021). proteins by machine learning and physics-based refinement. BioRxiv (2020).

Grünewald, F. et al. Polyply: a python suite for facilitating simulations of (bio-) macromolecules and nanomaterials. arXiv preprint arXiv:2105.05890 (2021). (2011).

Corradi, V. et al. Lipid-protein interactions are unique fingerprints for membrane proteins. ACS central science 4, 709-717 (2018).

Buyan, A. et al. Piezo1 forms specific, functionally important interactions with phosphoinositides and cholesterol. Biophysical journal 119, 1683-1697 (2020).

Ingólfsson, H. I. et al. Lipid organization of the plasma membrane. Journal of the american chemical society 136, 14554-14559 (2014).

Pezeshkian, W., König, M., Marrink, S. J. \& Ipsen, J. H. A multi-scale approach to membrane remodeling processes. Frontiers in molecular biosciences 6, 59 (2019).

Ramakrishnan, N., Kumar, P. S. \& Ipsen, J. H. Membrane-mediated aggregation of curvatureinducing nematogens and membrane tubulation. Biophysical journal 104, 1018-1028 (2013).

Pezeshkian, W., König, M., Wassenaar, T. A. \& Marrink, S. J. Backmapping triangulated surfaces to coarse-grained membrane models. Nature communications 11, 1-9 (2020). performance using a shorter cutoff and GPUs. Computer Physics Communications 199, 1-7 (2016).

Wassenaar, T. A., Ingólfsson, H. I., Bo $\square$ ckmann, R. A., Tieleman, D. P. \& Marrink, S. J. Computational lipidomics with insane: a versatile tool for generating custom membranes for molecular simulations. Journal of chemical theory and computation 11, 2144-2155 (2015).

\section{6 (1986).}

Shinoda, W., Namiki, N. \& Okazaki, S. Molecular dynamics study of a lipid bilayer: convergence, structure, and long-time dynamics. The Journal of chemical physics 106, 5731-5743 (1997).

Grieshammer, S., Belova, I. V. \& Murch, G. E. Thermodiffusion and ion transport in doped ceria by molecular dynamics simulations. Acta Materialia 210, 116802 (2021).

Wassenaar, T. A., de Vries, S., Bonvin, A. M. \& Bekker, H. SQUEEZE-E: The optimal solution for molecular simulations with periodic boundary conditions. Journal of chemical theory and computation 8, 3618-3627 (2012).

Greene, L. H. \& Higman, V. A. Uncovering network systems within protein structures. Journal of molecular biology 334, 781-791 (2003).

Seeber, M. et al. Wordom: a user $\square$ friendly program for the analysis of molecular structures, trajectories, and free energy surfaces. Journal of computational chemistry 32, 1183-1194 (2011).

Seeber, M., Felline, A., Raimondi, F., Mariani, S. \& Fanelli, F. WebPSN: a web server for highthroughput investigation of structural communication in biomacromolecules. Bioinformatics $\mathbf{3 1}$, 779-781 (2015).

Lata, S. \& Akif, M. Comparative Protein Structure Network Analysis on 3CLpro from SARS $\square$ CoV $\square 1$ and SARS $\square \operatorname{CoV} \square$ 2. Proteins: Structure, Function, and Bioinformatics (2021). Yen, H.-Y. et al. PtdIns $(4,5)$ P 2 stabilizes active states of GPCRs and enhances selectivity of Gprotein coupling. Nature 559, 423-427 (2018). 
49 Hedger, G. et al. Cholesterol interaction sites on the transmembrane domain of the hedgehog signal transducer and class FG protein-coupled receptor smoothened. Structure 27, 549-559. e542 (2019).

50 Hsu, P.-C., Jefferies, D. \& Khalid, S. Molecular dynamics simulations predict the pathways via which pristine fullerenes penetrate bacterial membranes. The journal of physical chemistry $B \mathbf{1 2 0}$, 11170-11179 (2016).

51 Hendrickx, J. M., Jungers, R. M., Olshevsky, A. \& Vankeerberghen, G. Graph diameter, eigenvalues, and minimum-time consensus. Automatica 50, 635-640 (2014).

52 Orman, K., Labatut, V. \& Cherifi, H. in Complex Networks 99-110 (Springer, 2013).

53 Barrat, A., Barthelemy, M., Pastor-Satorras, R. \& Vespignani, A. The architecture of complex weighted networks. Proceedings of the national academy of sciences 101, 3747-3752 (2004).

54 Aho, K., Derryberry, D. \& Peterson, T. Model selection for ecologists: the worldviews of AIC and BIC. Ecology 95, 631-636 (2014). Python Software Foundation. <https://docs.python.org/3/>

56 Bokeh Development Team. Bokeh: Python library for interactive visualization, $<$ http://www.bokeh.pydata.org> (2018).

57 Matlab, V. 7.10. 0 (R2010a). The MathWorks Inc., Natick, Massachusetts (2010).

58 Liu, F., Choi, D., Xie, L. \& Roeder, K. Global spectral clustering in dynamic networks. Proceedings of the National Academy of Sciences 115, 927-932 (2018).

59 Humphrey, W., Dalke, A. \& Schulten, K. VMD: visual molecular dynamics. Journal of molecular graphics 14, 33-38 (1996).

60 Stone, J. An Efficient Library for Parallel Ray Tracing and Animation, Citeseer, (1998).

61 The ImageMagick Development Team. ImageMagick, < https://imagemagick.org> 